\title{
SIMPLE AND SEMISIMPLE NEAR-RINGS
}

\section{W. BLACKETT}

Algebraic systems with addition and multiplication which satisfy all of the skew-field axioms except possibly one of the distributive laws have been studied occasionally. Dickson [1] gave examples which showed that such systems-called Fastkörper by Zassenhauscan actually be nondistributive. Zassenhaus [2] proved that Dickson's examples include all but seven of the finite Fastkörper which are not skew-fields. All continuous Fastkörper of finite degree over the reals were found by Kalscheuer [3]. Reidemeister [4] connected Fastkörper with the geometry of webs. All these results are, in a certain sense, extensions of well known facts about fields. This paper makes an analogous extension of part of the theory of semisimple rings to semisimple "Fastringe" or "near-rings."

Definition. A set $N$ of elements which can be added and multiplied is said to form a near-ring if

1. the elements form a group under addition,

2. the multiplication of elements is associative,

3. $n_{1}\left(n_{2}+n_{3}\right)=n_{1} n_{2}+n_{1} n_{3}$ for any selection of elements $n_{1}, n_{2}$, and $n_{3}$ from $N$.

It follows from this definition that a near-ring satisfies all of the usual ring axioms with the possible exceptions of the right distributive law and the commutative law of addition.

The most natural example of near-rings is given by the mappings of a group (written additively) into itself. If the mappings are added by adding images and multiplied by iteration they form a near-ring. ${ }^{1}$

A near-ring homomorphism is a mapping $\tau$ of a near-ring $N$ into a near-ring $N^{\prime}$ such that

$$
\left(n_{1}+n_{2}\right) \tau=n_{1} \tau+n_{2} \tau \text { and }\left(n_{1} n_{2}\right) \tau=\left(n_{1} \tau\right)\left(n_{2} \tau\right) \text {. }
$$

The two-sided ideals of a near-ring $N$ are defined to be the kernels of homomorphisms of $N$. The kernel $K$ of a near-ring homomorphism $\tau$ of $N$ is an additive normal subgroup of $N$. Since near-rings satisfy the left distributive law,

Presented to the Society, September 3, 1952; received by the editors March 6, 1953.

${ }^{1}$ For a more complete description of this example see H. Zassenhaus, Lehrbuch der Gruppentheorie, Berlin, 1937, pp. 71-74, or N. Jacobson, The theory of rings, New York, American Mathematical Society, 1943, pp. 1-2. 


$$
n 0=0
$$

when $n$ is any element of $N$ and 0 is the additive identity of $N$. Therefore $N K \subset K$. Also

$$
\begin{aligned}
{\left[\left(n_{1}+k\right) n_{2}-n_{1} n_{2}\right] \tau } & =\left[\left(n_{1}+k\right) n_{2}\right] \tau-\left(n_{1} n_{2}\right) \tau \\
& =\left[\left(n_{1}+k\right) \tau\right]\left(n_{2} \tau\right)-\left(n_{1} n_{2}\right) \tau \\
& =\left(n_{1} \tau+k \tau\right)\left(n_{2} \tau\right)-\left(n_{1} n_{2}\right) \tau \\
& =\left(n_{1} \tau\right)\left(n_{2} \tau\right)-\left(n_{1} \tau\right)\left(n_{2} \tau\right) \\
& =0
\end{aligned}
$$

for $n_{1}$ and $n_{2}$ in $N$ and $k$ in $K$. Thus $\left(n_{1}+k\right) n_{2}-n_{1} n_{2}$ is in $K$ for every selection $n_{1}$ and $n_{2}$ from $N$ and $k$ from $K$.

Suppose now that $H$ is any additive normal subgroup of $N$ such that $N H \subset H$ and $\left(n_{1}+h\right) n_{2}-n_{1} n_{2}$ is in $H$ for $n_{1}$ and $n_{2}$ in $N$ and $h$ in $H$. If $\sigma$ is the natural group homomorphism of $N$ onto $N-H$, a simple calculation shows that the definition

$$
\left(n_{1}+H\right)\left(n_{2}+H\right)=n_{1} n_{2}+H
$$

makes $N-H$ a near-ring and allows $\sigma$ to be considered a near-ring homomorphism.

Thus the two-sided ideals of $N$ are just the additive normal subgroups $T$ of $N$ such that

1. $N T \subset T$,

2. $\left(n_{1}+t\right) n_{2}-n_{1} n_{2}$ is in $T$ if $n_{1}$ and $n_{2}$ are in $N$ and $t$ is in $T$.

An additive group $G$ with the elements of $N$ as right operators is a right $N$-group if

$$
\left(g n_{1}\right) n_{2}=g\left(n_{1} n_{2}\right)
$$

for every selection of $n_{1}$ and $n_{2}$ from $N$ and $g$ from $G$. If "operating by $n^{n}$ is defined as right multiplication by $n$, a near-ring $N$ is itself a right $N$-group. The additive subgroups of $N$ which are also right $N$-groups are called the right modules of $N$. These modules are just the subgroups $M$ such that $M N \subset M$.

A group homomorphism $\tau$ of a right $N$-group $G$ into a right $N$ group is a right $N$-homomorphism if

$$
(g n) \tau=(g \tau) n
$$

for every $g$ and $n$.

The right ideals of $N$ are defined to be the kernels of homomorphisms of $N$ regarded as a right $N$-group. A check shows that the right ideals are just the additive normal subgroups $R$ of $N$ with

$$
\left(r+n_{1}\right) n_{2}-n_{1} n_{2} \in R
$$


for every $n_{1}, n_{2}$, and $r$.

For a ring the set of right modules is identical with the set of right ideals. This is not true in general for near-rings. The near-ring of all mappings of a finite group (with order greater than two) into itself is an example where the whole near-ring $N$ is the only right ideal which is also a right module. Consideration will shortly be restricted to those near-rings such that $0 n=0$ for all $n$. For these near-rings the set of right modules contains the set of right ideals.

Left $N$-groups and left $N$-homomorphisms may be defined analogously. If "operating by $n$ " is left multiplication by $n, N$ is a left $N$-group. The left ideals of $N$ are defined to be the kernels of homomorphisms of $N$ considered as a left $N$-group. These ideals are just the additive normal subgroups $L$ of $N$ with $N L C L$. In terms of these definitions, a two-sided ideal of $N$ is just a right ideal which is also a left ideal.

A right $N$-group $V$ is called a right representation space of $N$ or simply a right $N$-space if

$$
v\left(n_{1}+n_{2}\right)=v n_{1}+v n_{2}
$$

for every $n_{1}, n_{2}$, and $v$. A right $N$-space is proper if $V N \neq 0$. An irreducible right $N$-space is defined as a proper $N$-space with no subspaces other than the whole space and the space containing only 0 . The right modules of $N$ are examples of right $N$-spaces. Since a right $N$-homomorphism of a right $N$-space $V$ automatically preserves addition of operators, representation space homomorphisms are just the right $N$-homomorphisms which carry one representation space into another.

The sets of left $N$-groups, right $N$-groups, right modules, left ideals, right ideals, two-sided ideals, and right representation spaces of a near-ring $N$ are all closed under finite intersection. If the sum of two left, right, or two-sided ideals is defined as the set of all sums with a summand from each of the ideals, the sets of left, right, and twosided ideals are closed under finite sums. This is not necessarily true of the right modules. An $N$-group, an ideal, or a representation space is a direct sum of other $N$-groups, ideals, or representation spaces if it is a direct sum of them when considered as an additive group.

A simple calculation shows that the elements of a near-ring $N$ which annihilate a right $N$-space $V$ form a two-sided ideal $A_{V}$ of $N$. Similarly the set of elements of $N$ annihilating one particular element $v$ of $V$ is a right ideal $A_{v}$.

From this point on all the near-rings considered will be assumed to satisfy 
Axiom. $0 n=0$ for all $n$ in the near-ring.

Definition. A near-ring $N$ is semisimple if it has no nonzero nilpotent right modules and the right modules satisfy the descending chain condition.

THEOREM 1. Every nonzero module of a semisimple near-ring $N$ contains a nonzero idempotent. Every minimal nonzero right module $M$ is an irreducible $N$-space and contains an idempotent e such that

$$
e N=e M=M .
$$

Because the descending chain condition is satisfied by the right modules of $N$, any nonzero module contains a minimal nonzero module. Therefore, the first statement of the theorem is a consequence of the second. If $M$ is a minimal nonzero module, $M M \neq 0$ since $N$ contains no nilpotent nonzero modules. This shows that $M$ is a proper $N$-space and hence an irreducible $N$-space. Since $M^{2} \neq 0$, there is an element $m_{0}$ of $M$ such that $m_{0} M \neq 0$. The mapping $\tau: m \rightarrow m_{0} m$ of $M$ into itself is an $N$-homomorphism under which the image of $M$ is not zero. The kernel of the homomorphism is a right module because of the assumption $0 n=0$ for all $n$. The minimal property of $M$ implies that $m_{0} M=M$ and that $A_{m_{0}} \cap M$ is zero. Therefore $\tau$ is an $N$-isomorphism of the module $M$ onto itself. Now there is some element $e$ in $M$ such that $m_{0} e=m_{0}$. Hence, $m_{0} e e=m_{0} e$. It follows that $e^{2}=e$ because $\tau$ is an isomorphism. Now $e M \subset e N \subset M$ because $e \in M$. Since the module $e M$ contains the nonzero element $e$ of the irreducible space $M, M \subset e M$. Therefore $e M=e N=M$.

It is to be noted that if $e$ is any idempotent and $x$ is an element of $e N$, then $e x=x$. This follows because $x=e n$ implies $e x=e e n=e n=x$.

Theorem 2. A minimal nonzero right ideal $R$ of a semisimple nearring $N$ is an irreducible right representation space of $N$.

Because $0 n=0$ for all $n$ in $N, R$ is a right module. If $M$ is some minimal nonzero module contained in $R$ and $m_{0}$ is some element of $M$ such that $m_{0} M \neq 0$, then the mapping $\tau: n \rightarrow m_{0} n$ is an $N$-homomorphism of $N$ as a right $N$-group onto $M$. The kernel of $\tau$ is the right ideal $A_{m_{0}}$ of annihilators of $m_{0}$. Since $R$ is a minimal right ideal, either $R \cap A_{m_{0}}=R$ or $R \cap A_{m_{0}}=0$. Now $R \supset M$, but $A_{m_{0}} D M$. Hence $R \cap A_{m_{0}}=0$. Therefore $\tau$ restricted to $R$ is an $N$-isomorphism of $R$ onto $M$. But $M \tau=M$. Hence, $R=M$. By Theorem $1, M$ is an irreducible $N$-space.

TheOREM 3. A nonzero right module $M$ of a semisimple near-ring $N$ is a finite direct sum of minimal nonzero modules contained in it. 
By the descending chain condition, $M$ contains a minimal nonzero module $M_{0}$. Theorem 1 shows that an idempotent element $e_{0}$ may be chosen such that $e_{0} N=e_{0} M_{0}=M_{0}$. If $m$ is any element of $M$,

$$
m=e_{0} m+\left(-e_{0} m+m\right) \text {. }
$$

Here $e_{0} m$ is in $M_{0}$ and $-e_{0} m+m$ is in $A_{e_{0}}$. The latter follows because

$$
e_{0}\left(-e_{0} m+m\right)=-e_{0}^{2} m+e_{0} m=-e_{0} m+e_{0} m=0 .
$$

Therefore $M \subset M_{0}+A_{e_{0}}$. Also $M_{0} \cap A_{e_{0}}=0$ because $e_{0} m_{0}=m_{0}$ for $m_{0}$ in $M_{0}$. If $M=M_{0}$, the theorem is proved. If $M \neq M_{0}$, then $M \supset M \cap A_{\sigma_{0}}$ $\neq 0$, that is, $M$ properly contains a nonzero submodule which is the intersection of $M$ with a right ideal of $N$. Similarly either $M \cap A_{e_{0}}$ is a minimal nonzero module or it properly contains a smaller nonzero submodule of the form $\left(M \cap A_{e_{0}}\right) \cap R$ where $R$ is a right ideal of $N$. Since $A_{e_{0}} \cap R$ is a right ideal of $N, M \cap\left(A_{e_{0}} \cap R\right)$ is the intersection of $M$ with a right ideal of $N$. Because of the descending chain condition on right modules this process must lead after a finite number of steps to a minimal nonzero module $M_{1}$ which is the intersection of $M$ with a right ideal of $N$. In particular this means that $M_{1}$ is an additive normal subgroup of $M$. After the selection of an idempotent $e_{1}$ in $M_{1}$, such that $e_{1} N=e_{1} M_{1}=M_{1}, M$ can be written as the direct sum $M_{1} \oplus\left(M \cap A_{e_{1}}\right)$, because both $M_{1}$ and $\left(M_{1} \cap A_{\epsilon_{1}}\right)$ are normal subgroups of $M$. Repetition of the procedure with $\left(M_{1} \cap A_{e_{1}}\right)$ and succeed-

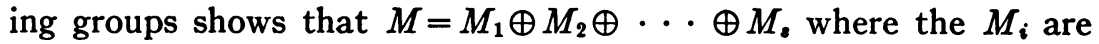
minimal nonzero modules contained in $M$. The chain condition assures that this decomposition is of finite length.

If the additive group of $N$ is abelian, the module $M_{0}$ could serve as a starting point of the decomposition, and the proof of the theorem would have been simplified.

CoROllary 1. A nonzero right ideal $R$ of a semisimple near-ring $N$ is the direct sum of minimal nonzero right ideals contained in it.

If the module $M$ in the proof of the theorem happens to be a right ideal, $M_{1}, M_{2}, \cdots, M_{8}$ are also right ideals.

This remark, which proves the corollary, also gives a new proof of Theorem 2.

THEOREM 4. Every right ideal $R$ of a semisimple near-ring $N$ contains an idempotent $e$ such that $e R=R$.

The procedure used in proving Theorem 3 allows the assumption that $R$ is represented as the direct sum $R=e_{1} R \oplus \cdots \oplus e_{p} R$ where $e_{i}$ 
is an idempotent, $e_{i} R=e_{i} N$ is a minimal right ideal, and $e_{i} R \oplus \ldots$ $\oplus e_{p} R$ is in $A_{e_{i-1}}$.

First it will be proved by induction that

$$
\left(e_{1}+e_{2}+\cdots+e_{p}\right) R=e_{1} R \oplus e_{2} R \oplus \cdots \oplus e_{p} R=R .
$$

Assume as an induction hypothesis that

$$
\left(e_{1}+\cdots+e_{8}\right) R=e_{1} R \oplus \cdots \oplus e_{8} R .
$$

Since the summands $e_{i} R$ of $R$ are all right ideals,

$$
\left(e_{1}+\cdots+e_{8}+e_{8+1}\right) r-\left(e_{1}+\cdots+e_{8}\right) r
$$

is in $e_{a+1} R$ and

$$
\left(e_{1}+\cdots+e_{8}+e_{8+1}\right) r-e_{8+1} r
$$

is in $e_{1} R \oplus \cdots \oplus e_{8} R_{8}$. Therefore

$$
\left(e_{1}+\cdots+e_{s}+e_{2+1}\right) r-\left(e_{1}+\cdots+e_{2}\right) r-e_{s+1} r
$$

is in

$$
\left(e_{1} R+\cdots+e_{8} R\right) \cap e_{s+1} R .
$$

Because this intersection contains only zero,

$$
\left(e_{1}+\cdots+e_{8}+e_{8+1}\right) r=\left(e_{1}+\cdots+e_{8}\right) r+e_{8+1} r .
$$

Repetition of this argument shows that

$$
\left(e_{1}+\cdots+e_{s}+e_{s+1}\right) r=e_{1} r+\cdots+e_{s} r+e_{s+1} r
$$

for any element $r$ of $R$.

Now

$$
\left(e_{1}+\cdots+e_{8}+e_{8+1}\right) e_{8+1} r=e_{8+1} r
$$

because $e_{s+1} R$ is in $A_{e_{i}}$ for $i \leqq s$. Therefore $\left(e_{1}+\cdots+e_{s+1}\right) R$ contains $e_{s+1} R$.

On the other hand

$$
\begin{aligned}
\left(e_{1}+\cdots+e_{s+1}\right)(r- & \left.e_{8+1} r\right) \\
& =\left(e_{1}+\cdots+e_{s+1}\right) r-\left(e_{1}+\cdots+e_{s+1}\right) e_{8+1} r \\
& =\left(e_{1}+\cdots+e_{8}\right) r+e_{s+1} r-e_{s+1} r \\
& =\left(e_{1}+\cdots+e_{s}\right) r .
\end{aligned}
$$

Hence $\left(e_{1}+\cdots+e_{s+1}\right) R$ contains $\left(e_{1}+\cdots+e_{s}\right) R$.

These facts, combined with the assumption that 


$$
\left(e_{1}+\cdots+e_{8}\right) R=e_{1} R \oplus \cdots \oplus e_{8} R
$$

prove that

$$
\left(e_{1}+\cdots+e_{s+1}\right) R=e_{1} R \oplus \cdots \oplus e_{s+1} R .
$$

Since $e_{1} R=e_{1} R$, it follows by induction that

$$
\left(e_{1}+\cdots+e_{p}\right) R=e_{1} R \oplus \cdots \oplus e_{p} R=R .
$$

It now follows that the right homomorphism of $N$ defined by $\tau: n \rightarrow\left(e_{1}+\cdots+e_{p}\right) n$ carries $R$ onto itself.

If

$$
r=r_{s}+r_{s+1}+\cdots+r_{p} \quad\left(r_{i} \in e_{i} R\right)
$$

is an element of $R$ such that $r_{s} \neq 0$, then

$$
\begin{aligned}
\left(e_{1}+\cdots+\right. & \left.+e_{p}\right) r \\
= & \left(e_{1}+\cdots+e_{p}\right)\left(r_{s}+\cdots+r_{p}\right) \\
= & e_{1}\left(r_{s}+\cdots+r_{p}\right)+\cdots+e_{s}\left(r_{s}+\cdots+r_{p}\right)+\cdots \\
& +e_{p}\left(r_{s}+\cdots+r_{p}\right) \\
= & 0+e_{s}\left(r_{s}+\cdots+r_{p}\right)+e_{s+1}\left(r_{s}+\cdots+r_{p}\right)+\cdots \\
& +e_{p}\left(r_{s}+\cdots+r_{p}\right) \\
= & e_{s} r_{s}+e_{s}\left(r_{s+1}+\cdots+r_{p}\right)+e_{s+1}\left(r_{s}+\cdots+r_{p}\right)+\cdots \\
& +e_{p}\left(r_{s}+\cdots+r_{p}\right) \\
= & e_{s} r_{s}+e_{s+1}\left(r_{s}+\cdots+r_{p}\right)+\cdots+e_{p}\left(r_{s}+\cdots+r_{p}\right) .
\end{aligned}
$$

Since $e_{8} r_{s}=r_{8} \neq 0$ and $\left(e_{1}+\cdots+e_{p}\right) r-e_{s} r_{8}$ is in $e_{s+1} R \oplus \cdots \oplus e_{p} R$,

$$
\left(e_{i}+\cdots+e_{p}\right) r \neq 0 \text {. }
$$

This proves that $\tau$ acts as an automorphism on $R$. If $e$ is the element of $R$ such that

$$
\left(e_{1}+\cdots+e_{p}\right) e=e_{1}+\cdots+e_{p}
$$

then $e^{2}=e$, and $e R=R$. This proves the theorem.

COROLlaRY 1. Every semisimple near-ring has a left unit.

By Theorem 4 the near-ring has a generating idempotent. On the other hand, any idempotent $e$ is a left unit for $e N$.

Corollary 2. If $R=R_{1} \oplus \cdots \oplus R_{p}$ is a direct sum decomposition of a right ideal $R$ into smaller right ideals $R_{i}$, generating idempotents $e_{i}$ can be selected from the respective $R_{i}$ so that $e_{i} e_{j}=0$ for $i \neq j$. 
By Theorem 4, $R$ has an idempotent $e$ such that $e R=R$. This idempotent, which must act as a left unit on $R$, has a decomposition $e=e_{1}+\cdots+e_{p}\left(e_{i} \in R_{i}\right)$. As in the proof of Theorem 4,

$$
r_{i}=e r_{i}=\left(e_{1}+\cdots+e_{p}\right) r_{i}=e_{1} r_{i}+\cdots+e_{p} r_{i}
$$

for any $r_{i}$ in $R_{i}$. This shows that $e_{j} r_{i}=0$ for $j \neq i$ and that $e_{i} r_{i}=r_{i}$.

COROLLARY 3. The right ideals of a semisimple near-ring satisfy the ascending chain condition.

Suppose $R_{1} \subset R_{2} \subset R_{3} \subset \cdots$ is an ascending chain of distinct right ideals. Let $R=U_{i} R_{i}$. Select idempotents $e_{i}$ such that $e_{i} \in R_{i}$ and $e_{i} R_{i}=R_{i}$. Now $R_{i+1}=R_{i} \oplus\left(R_{i+1} \cap A_{e_{i}}\right)$. Therefore

$$
R=R_{i} \oplus R_{i}^{\prime}
$$

where

$$
R_{i}^{\prime}=\left(R_{i+1} \cap A_{e_{i}}\right) \oplus\left(R_{i+2} \cap A_{e_{i+1}}\right) \oplus \cdots \cdot
$$

Since the properly descending chain

$$
R_{1}^{\prime} \supset R_{2}^{\prime} \supset R_{\mathbf{z}}^{\prime} \supset \cdots
$$

must be finite, the original ascending chain must also be finite.

THEOREM 5. If $N$ is a semisimple near-ring and $V$ is an irreducible right $N$-space, $V$ is $N$-isomorphic to a minimal nonsero right ideal of $N$. In particular $V$ is $N$-isomorphic to any minimal nonzero right ideal $R$ for which $V R \neq 0$.

Because irreducible spaces are proper, $V N \neq 0$. Therefore it follows from Corollary 1 of Theorem 3 applied to $N$ that $V R \neq 0$ for some minimal nonzero right ideal $R$. If $v$ is an element of $V$ such that $v R \neq 0$, the mapping $\tau: r \rightarrow v r$ is an $N$-homomorphism of $R$ into $V$. Since $V$ is irreducible, $\tau$ maps $R$ onto $V$. The kernel $R \cap A_{v}$ of $\tau$ is 0 because $R$ is a minimal nonzero right ideal. Hence $V$ is $N$-isomorphic to $R .^{2}$

TheOREM 6. A semisimple near-ring has only a finite number of non- $N$-isomorphic types of irreducible spaces. The number of types is the same as the number of types of minimal nonzero right ideals which are not $N$-isomorphic.

Theorems 2 and 5 show that the number of types of irreducible spaces is the same as the number of types of minimal nonzero ideals. It only remains to prove that this number is finite. The common

2 A suggestion of the referee simplified this proof. 
proofs of the Jordan-Hölder Theorem for groups with linear operators carry over almost verbatim to proofs of the analogous theorem for right ideals of a semisimple near-ring. This theorem allows the normal series $N \supset R \supset 0$ ( $R$ a nonzero right ideal) to be refined to a finite composition series. If $R$ is a minimal right ideal, it must be a factor of this composition series. Since all the composition series have the same factors, the number of non- $N$-isomorphic types of minimal right ideals is finite.

Definition. A nonzero near-ring $N$ is simple if

1. it has no proper two-sided ideals,

2. its right modules satisfy the descending chain condition,

3. it has no nonzero right modules which are annihilated from the right by all the elements of $N$.

TheOREм 7. A simple near-ring is semisimple and has one and only one type of irreducible space.

By the definition of simplicity, the right modules satisfy the descending chain condition. If $M$ is a nonzero nilpotent module, there is some integer $k$ such that $M^{k}=0$ but $M^{k-1} \neq 0$. Therefore, the twosided ideal $A_{M}$ of annihilators of $M$ contains $M^{k-1} \neq 0$. This implies that $A_{M}=N$, which contradicts Condition 3 above. Thus $N$ is a semisimple near-ring. Since $N$ is not the near-ring consisting of zero alone, $N$ must have a minimal nonzero module and hence at least one type of irreducible $N$-space. If $V_{1}$ and $V_{2}$ are two irreducible $N$-spaces, neither of them can be annihilated by a minimal right ideal $R$ of $N$, because the two-sided ideal of annihilators of either $V_{1}$ or $V_{2}$ must be the zero ideal. By Theorem 5 both $V_{1}$ and $V_{2}$ are $N$-isomorphic to $R$ and hence to each other.

THEOREM 8. A semisimple near-ring $N$ with exactly one type of irreducible $N$-space is a simple near-ring.

The right modules satisfy the descending chain condition because $N$ is semisimple. There can be no nonzero right module annihilated by $N$ since such a module would satisfy the equation $M^{2}=0$. If $T$ is a proper two-sided ideal of $N$, there is at least one minimal right ideal not contained in $T$, because a semisimple near-ring is the direct sum of some of its minimal right ideals. If $R$ is such a right ideal, $R \cap T=0$ because $R$ is minimal. Also $R T \subset R \cap T$. Hence $R T=0$. Now $R$ and any minimal right ideal $R^{\prime}$ contained in $T$ are $N$-isomorphic because they are both irreducible $N$-spaces. Therefore $R^{\prime} R^{\prime} \subset R^{\prime} T=0$, and $R^{\prime}$ is nilpotent. This contradiction shows that $N$ is simple.

For the rest of this paper the term "semisimple near-ring" will be 
interpreted so as to exclude the trivial near-ring with only one element.

If $N$ is a semisimple near-ring, Theorem 6 permits the choice of non- $N$-isomorphic irreducible $N$-spaces $V_{1}, \cdots, V$, so that any other irreducible $N$-space is $N$-isomorphic to one of the $V_{i}$. After such a selection, a two-sided ideal $A_{i}^{0}$ corresponding to $V_{i}$ may be defined by

$$
A_{i}^{0}=\bigcap_{j \neq i} A_{\nabla_{j}}
$$

Cleariy this definition depends only on the type of irreducible space and not on the particular choice of the representative $V_{i}$.

LEMмA 1: $A$ minimal right module is contained in $A_{i}^{0}$ if and only if it is $N$-isomorphic to $V_{i}$.

A minimal right module $M$ which is $N$-isomorphic to $V_{j}$, where $j \neq i$, is annihilated by $A_{i}^{0}$ since $A_{i}^{0} \subset A_{V_{j}}$. Therefore, $M$ cannot be contained in $A_{\imath}^{0}$ because $M$ would then be nilpotent. If $M$ is a minimal module $N$-isomorphic to $V_{i}, V_{j} M=0$ for $j \neq i$. This is the case, for otherwise the mapping $m \rightarrow v ; m$ would be an $N$-isomorphism of $M$ onto $V_{j}$ for any $v_{j}$ such that $v_{j} M \neq 0$. This shows that $M \subset A_{\nabla_{j}}$ for all $j \neq i$. Therefore $M \subset A_{i}^{0}$.

Theorem 9. $A_{i}^{0}$ is the sum of the modules $N$-isomorphic to $V_{i}$.

This follows immediately from Theorem 3 or its corollary, and Lemma 1.

LEMMA 2.

$$
\begin{aligned}
A_{i}^{0} \cap\left(\sum_{j \neq i} A_{j}^{0}\right) & =0, \\
\left(\sum_{j \neq i} A_{j}^{0}\right) A_{i}^{0} & =0,
\end{aligned}
$$$$
i=1,2, \cdots, s \text {. }
$$

From the definition of $A_{k}^{0}$,

$$
A_{i}^{0} \cap \sum_{j \neq i} A_{j}^{0}=\bigcap_{k=1}^{0} A_{V_{k}}
$$

If $\bigcap_{k=1}^{k} A_{V_{k}}$ were not the zero ideal, it would contain a minimal nonzero right ideal. This ideal would be $N$-isomorphic to one of the $V_{k}$ and accordingly would be nilpotent. Therefore

$$
A_{i}^{0} \cap \sum_{j \neq i} A_{j}^{0}=0
$$


Since $\sum_{j \neq i} A_{j}^{0}$ and $A_{i}^{0}$ are two-sided ideals,

$$
\left(\sum_{j \neq i} A_{j}^{0}\right) A_{i}^{0} \subset A_{i}^{0} \cap\left(\sum_{j \neq i} A_{j}^{0}\right) .
$$

THEOREM 10. The semisimple near-ring $N$ is the direct sum of the two-sided ideals $A_{\uparrow}^{0}$.

$N$ is the direct sum of minimal modules according to Theorem 3 . Lemma 1 shows that each of these modules is contained in some one of the $A_{i}^{0}$. Hence $N$ is the sum of the $A_{\uparrow}^{0}$. It follows from Lemma 2 that this sum is direct.

Theorem 11. The two-sided ideal $A_{i}^{0}$ of the semisimple near-ring $N$ is simple when it is considered as a near-ring.

If $M_{i}$ is a right $A_{i}^{0}$ module,

$$
\begin{aligned}
M_{i} N & =M_{i}\left(A_{1}^{0} \oplus \cdots \oplus A_{i}^{0}\right) \\
& =M_{i} A_{1}^{0}+\cdots+M_{i} A_{i}^{0} \\
& =M_{i} A_{i}^{0} \subset M_{i} .
\end{aligned}
$$

This shows that a right $A_{i}^{0}$-module is also a right $N$-module. If $M$ is an $N$-module, $M \cap A_{i}^{0}$ is both an $N$ - and an $A_{i}^{0}$-module. Therefore the minimal $A_{i}^{0}$-modules are just the minimal $N$-modules contained in $A_{\uparrow}^{0}$. This fact, combined with Lemma 1 , shows that $A_{i}^{0}$ as a nearring has one and only one type of irreducible $A_{i}^{0}$-space. A nilpotent $A_{i}^{0}$-module would be a nilpotent $N$-module. Also, a descending chain of $A_{i}^{0}$-modules can be regarded as a chain of $N$-modules. Therefore, the semi-simplicity of $N$ implies that $A_{\imath}^{0}$ is semisimple. The conclusion that $A_{i}^{0}$ is a simple near-ring now follows from Theorem 8 .

THEOREM 12. If $B$ is a nonzero two-sided ideal of the semisimple near$\operatorname{ring} N$,

$$
B=A_{i_{1}}^{0} \oplus \cdots \oplus A_{i i}^{0}
$$

for suitable $i_{k}$.

A given nonzero element $b$ of $B$ can be represented uniquely in the form

$$
b=a_{j_{1}}+\cdots+a_{j_{p}}
$$

where $a_{j_{k}}$ is in $A_{j_{k}}$ and $a_{j_{k}} \neq 0$. Because $A_{j_{k}}^{0}$ is simple, it can have no nonzero annihilators when considered as $A_{j_{k}}^{0}$-space. Therefore $A_{j_{k}}^{0} a_{j_{k}}$ $\neq 0$. Since 


$$
B \supset A_{j_{k}}^{0}\left(a_{j_{1}}+\cdots+a_{j_{p}}\right)=A_{j_{k}}^{0} a_{j_{k}} \neq 0, \quad A_{j_{k}}^{0} \cap B \neq 0 .
$$

Since $A_{j_{k}}^{0} \cap B$ is a two-sided ideal of $N$,

$$
A_{j_{k}}^{0} \cap B=A_{j_{k}}^{0} \text {. }
$$

Therefore

$$
B \supset A_{j_{1}}^{0} \oplus \cdots \oplus A_{j_{p}}^{0} \text {. }
$$

If $i_{k}$ runs through all the different indices $j_{k}$ which occur for some $b$ in $B$, then

$$
B=A_{i_{1}}^{0} \oplus \cdots \oplus A_{i s \cdot}^{0}
$$

CoRollary. If $N=B_{1} \oplus \cdots \oplus B_{i}$ where the $B_{i}$ are indecomposable two-sided ideals of the semisimple near-ring $N$, then $t=s$ and after rearrangement $B_{i}=A_{i}^{0}$.

ThEOREM 13. The semisimple near-ring $N$ is completely determined up to an isomorphism by the simple near-rings $A_{i}^{0}$.

Theorem 10 states that $N=A_{i}^{0} \oplus \cdots \oplus A_{s}^{0}$. Therefore the addition in $N$ is determined by the addition in the $A_{i}^{0}$. It only remains to be shown that the multiplication in $N$ is determined by the multiplication in the $A_{\imath}^{0}$. This will be proved as soon as it is shown that

$$
\left(a_{1}+\cdots+a_{8}\right)\left(a_{1}^{\prime}+\cdots+a_{s}^{\prime}\right)=a_{1} a_{1}^{\prime}+a_{2} a_{2}^{\prime}+\cdots+a_{8} a_{8}^{\prime}
$$

where $a_{i}$ and $a_{i}^{\prime}$ are in $A_{i}^{0}$. Because the $A_{i}^{0}$ are right ideals, and

$$
A_{i}^{0} \cap\left(\sum_{j \neq i} A_{j}^{0}\right)=0(\text { Lemma 2), }
$$

an argument analogous to the one used in the proof of Theorem 4 to show that

$$
\left(e_{1}+\cdots+e_{s+1}\right) r=e_{1} r_{1}+\cdots+e_{s+1} r
$$

proves that

$$
\begin{aligned}
\left(a_{1}+\cdots+a_{s}\right) & \left(a_{1}^{\prime}+\cdots+a_{s}^{\prime}\right) \\
& =a_{1}\left(a_{1}^{\prime}+\cdots+a_{s}^{\prime}\right)+\cdots+a_{s}\left(a_{1}^{\prime}+\cdots+a_{s}^{\prime}\right) .
\end{aligned}
$$

Use of the left distributive law gives

$$
\begin{aligned}
\left(a_{1}+\cdots+a_{s}\right) & \left(a_{1}^{\prime}+\cdots+a_{s}^{\prime}\right) \\
& =a_{1} a_{1}^{\prime}+\cdots+a_{1} a_{s}^{\prime}+\cdots+a_{s} a_{1}^{\prime}+\cdots+a_{s} a_{s}^{\prime} .
\end{aligned}
$$


But by Lemma 2, $a_{i} a_{j}^{\prime}=0$ for $i \neq j$.

THEOREM 14. If $N_{1}, N_{2}, \cdots$, and $N$, are simple near-rings, and $N$ is defined to be the set of s-tuples

$$
\left(n_{1}, \cdots, n_{s}\right), \quad n_{i} \in N_{i},
$$

then $N$ is a semisimple near-ring if the s-tuples are added and multiplied componentwise. If the types of irreducible spaces are suitably labeled, $A_{i}^{0}$ is composed of the s-tuples

$$
\left(0, \cdots, 0, n_{i}, 0, \cdots, 0\right)
$$

where $n_{i}$ is in $N_{i}$.

It is clear from the definition of $N$ that $N$ is a near-ring. If $M$ is a right $N$-module with elements

$$
m=\left(m_{1}, m_{2}, \cdots, m_{s}\right),
$$

the set $M_{i}$ of all elements $m_{i}$ of $N_{i}$ which occur as $i$ th components of some element of $M$ form a right $N_{i}$-module. Since $N$ contains all elements of the form

$$
\left(0, \cdots, 0, n_{i}, 0, \cdots, 0\right),
$$

it follows that $M$ contains the $N$-module $\left(M_{i} N_{i}\right)^{*}$ which consists of all elements of the form

$$
\left(0, \cdots, 0, m_{i} n_{i}, 0, \cdots, 0\right) .
$$

If $M_{i} \neq 0, M_{i}=M_{i 1} \oplus \cdots \oplus M_{i i_{k}}$ where each $M_{i j}$ is a minimal right $N_{i}$-module. Because $M_{i j}$ is minimal, $M_{i j} M_{i j}=M_{i j}$. Hence $M_{i j} N_{i}$ $=M_{i j}$ and $M_{i} N_{i}=M_{i}$. Therefore $M_{\supset} M_{i}^{*}$ where $M_{i}^{*}$ is the $N$-module with elements

$$
m_{i}^{*}=\left(0, \cdots, 0, m_{i}, 0, \cdots, 0\right) .
$$

Since $M_{i}^{*} \cap \sum_{j \neq i} M_{j}^{*}=0$ and each $M_{i}$ is a normal subgroup of $M$, $M=M_{1}^{*} \oplus \cdots \oplus M_{*}^{*}$. If $M \supset M^{\prime} \supset M^{\prime \prime} \supset \cdots$ is a descending chain of $N$-modules, it gives rise to corresponding chains $M_{i} \supset M_{i}^{\prime} \supset \ldots$ of $N_{i}$-modules. By the descending chain condition for right $N_{i}$ modules, each of these chains must stop properly descending after a finite number of terms. As soon as all of these chains have stopped decreasing, so also have the chains $M_{i}^{*} \supset M_{i}^{*} \supset \ldots$. The formula $M=M_{1}^{*} \oplus \cdots \oplus M_{*}^{*}$ now shows that the chain $M \supset M^{\prime} \supset M^{\prime \prime} \supset \cdots$ also stops decreasing. This proves that the right $N$-modules satisfy the descending chain condition. If $M$ is a nilpotent $N$-module, each of the $N_{i}$-modules $M_{i}$ is nilpotent. This implies that each $M_{i}=0$ 
$(i=1, \cdots, s)$. Hence $M=0$. This shows that $N$ is semisimple. The $N$-module $N_{i}^{*}$ is an indecomposable two-sided ideal of $N$. Since $N$ $=N_{1}^{*} \oplus \cdots \oplus N_{i}^{*}$, it follows from the corollary to Theorem 12 that, with suitable labeling, $A_{i}^{0}=N_{i}^{*}$.

\section{REFERENCES}

1. L. E. Dickson, On finite algebras, Nachr. Ges. Wiss. Gorttingen (1905) pp. 358393.

2. H. Zassenhaus, Über endliche Fastkörper, Abh. Math. Sem. Hamburgischen Univ. vol. 11 (1936) pp. 187-220.

3. F. Kalscheuer, Die Bestimmung aller stetigen Fastkörper, uber dem Körper der reellen Zahlen als Grundkörper, Abh. Math. Sem. Hamburgischen Univ. vol. 13 (1940) pp. 413-435.

4. K. Reidemeister, Grundlagen der Geometrie, Berlin, 1930, pp. 110-122.

Princeton University 